\section{Bakterielle Meningitiden eliminieren}

- Mit Impfstoffen gegen Hib, Pneumokokken und Meningokokken $\mathrm{C}$ ist der Anfang zur Ausrottung von Meningitiden bereits gemacht. Impfstoffe gegen weitere Meningokokken-Typen könnten bald die letzte große Lücke schließen, so Professor HeinzJosef Schmitt von Novartis Vaccines.

? Ein Schwerpunkt von Novartis Vaccines sind Meningokokken-Impfstoffe. Warum wird ein Impfschutz gegen diese Erreger so dringend gebraucht?

Schmitt: Mit knapp 500 Erkrankungen jährlich ist Meningokokken-Meningitis in Deutschland zwar relativ selten, aber gefürchtet: Rund 10\% der Patienten sterben und weitere $10 \%$ behalten schwere Schäden. Nicht selten sind Verläufe, in denen ein Kind oder ein Jugendlicher binnen 24 Stunden tot ist. In diesen Fällen helfen keine Antibiotika mehr, der Patient stirbt einem unter den Fingern weg.

? Novartis Vaccines hat jetzt mit Menveo ${ }^{\circledR}$ den ersten konjugierten MeningokokkenImpfstoff in Deutschland gegen die Serotypen ACWY auf den Markt gebracht. Bisher gab es Polysaccharid-Impfstoffe dagegen. Was sind die Vorteile des konjugierten Impfstoffs?

Schmitt: Die Polysaccharid-Impfstoffe sind nicht optimal, weil sie Säuglinge und Kleinkinder bis wenigstens zwei Jahre nicht effektiv schützen, weil die Schutzwirkung nur drei bis fünf Jahre reicht, weil das Immungedächtnis mit diesen Impfstoffen kaum aktiviert wird und weil daher der Impfschutz immer wieder erneuert werden muss. Aber genau das funktioniert nur schlecht, wegen der sogenannten „Hyporesponsiveness“, das heißt, bei einer wiederholten Impfung steigen die Antikörpertiter kaum noch an. Mit Konjugatimpfstoffen lassen sich hingegen auch Kinder im Alter unter zwei Jahren schützen. Das Immungedächtnis wird aktiviert, was zu einer deutlich längeren Schutzwirkung führt. Der Impfschutz lässt sich gut auffrischen auch bei weiteren Dosen, da es nicht zu einer Hyporesponsiveness kommt. Schließlich wird in der Bevölkerung die Trägerrate von Meningokokken reduziert. Dies führt bei hohen Impfraten zu einer Herdenimmunität. Kurz, Konjugatimpfstoffe bieten so viele Vorteile, dass es kaum noch vertretbar ist, Polysaccharid-Impfstoffe zu verwenden.

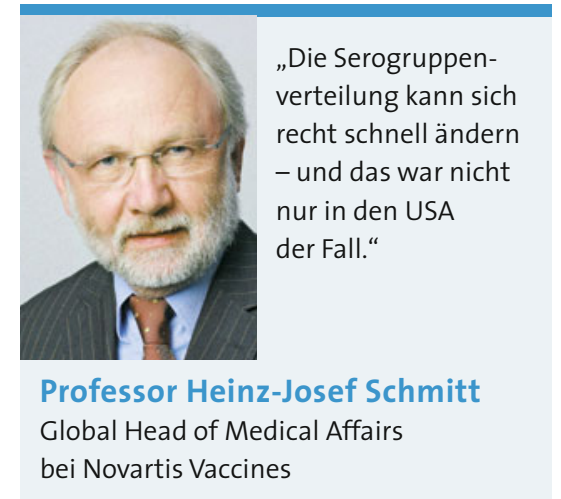

? Der neue Impfstoff schützt gegen die Serogruppen ACWY. Davon kommt in Deutschland nur die Serogruppe C häufig vor. Für welche Zielgruppen ist der neue viervalente Impfstoff gedacht?

Schmitt: Der neue Impfstoff sollte in Deutschland auch bei Aufenthalten in Endemiegebieten verwendet werden, etwa in Afrika. Für Hadsch- und Umra-Pilger ist eine Meningokokken-Impfung bei der Einreise nach Saudi-Arabien sogar vorgeschrieben. Außerdem profitieren Austauschschüler und -studenten zum Beispiel in die USA von dem Impfstoff. Dort hat sich der Anteil der Serogruppe $Y$ an den Infektionen zwischen 1990 und 2006 von 9 auf $39 \%$ erhöht. Hier sieht man, dass sich die Serogruppenverteilung recht schnell ändern kann - und das war nicht nur in den USA der Fall.

! Etwa $70 \%$ der Erkrankungen an Meningokokken-Meningitis werden in Deutschland durch die Gruppe B verursacht. Ihrem Unternehmen ist es jetzt mit einem neuen Verfahren gelungen, erstmals einen Impfstoff bis in Phase Ill der klinischen Prüfung zu bringen. Was ist das für ein Verfahren und was bringt der Impfstoff?

Schmitt: Sehr verkürzt dargestellt, wurden alle Proteine auf dem Genom von B-Meningokokken identifiziert - rund 500. Davon waren etwa $50 \mathrm{im}$ Tiermodell besonders immunogen, induzierten neutralisierende Antikörper und fanden sich außen am Bakterium. Drei der Proteine und ein sogenanntes „outer membrane vesicle " wurden schließlich für den Kandidat-Impfstoff ausgewählt. Erste Ergebnisse der Phase-III-Prüfung sind vielversprechend, und wir hoffen, die Daten bis Ende des Jahres zur Zulassung einreichen zu können.

\section{Maßgeschneiderte Tabletten}

Divergierende Leberstoffwechselsituationen, uneinheitliche Nierenfiltrationsvermögen und nicht-konforme Anwendungen von Wirkstoffen führen bei der Einnahme von Medikamenten zu individuellen Bedarfssituationen. Mit dem Dispetron-Verfahren ist es möglich geworden, einen weiteren Schritt in die personalisierte Therapie zu gehen. Die Tablette wird im Rahmen einer Rezeptur für den einzelnen Patienten mit Wirkstoff beladen, so kann die Dosis den individuellen Bedürfnissen entsprechend angepasst werden. Das Schmankerl für die Arzneimitteltherapie bei Kindern: Die Tabletten sind selbstsuspendierend und zerfallen auf einem Teelöffel in weniger als 120 Sekunden. Im Einsatz sind zurzeit die Wirkstoffe ASS, Phenprocoumon, Hydrocortison, Spironolacton, Clobazam und weitere. Der Bezug für Patienten erfolgt prompt über den Versand der VITA Apotheke in Hohen Neuendorf.

Nach Informationen von Laburnum $\mathrm{GmbH}$ Hohen Neuendorf

\section{Mit Folat angereicherte Pille gegen Neuralrohrdefekte}

Die Einnahme von Folaten bzw. Folsäure zur Prävention von Neuralrohrdefekten sollte bereits Wochen bis Monate vor der Konzeption beginnen. Da die obligate Anreicherung von Lebensmitteln in Deutschland keine Chance hat, stellt sich die Frage, wie eine perikonzeptionelle Substitution realisiert werden kann. Die Option heißt „PLUS-Konzept“ und besteht aus der Kombination eines hormonellen Kontrazeptivums mit einem Folat. Die Epidemiologie stützt dieses neue Konzept: Mehr als 20\% der Frauen werden nach dem Absetzen der Pille bereits im ersten Monat schwanger, nach drei Monaten sind es knapp $50 \%$. Haben diese Frauen zuvor mit einer Pille automatisch Folat substituiert, bleiben die Spiegel nach Absetzen für etwa drei Monate erhalten und bieten so einen wirksamen Schutz vor Neuralrohrdefekten. Die Zulassung für ein erstes Dienogest-Präparat plus 400 $\mu \mathrm{g}$ Folat wird für Anfang 2011 erwartet, im vierten Quartal 2011 sollen zwei drospirenonhaltige Produkte folgen. rb

Fachpressekonferenz „Das PLUS-Konzept mehr als Verhütung“, Berlin, 2. September 2010. Veranstalter: Bayer Vital, Leverkusen 